Vincent Genin, Incarner le droit international. La Belgique et ses juristes : du mythe juridique au déclassement international (1914-1940). Bruxelles-Bern-Berlin-New York-Oxford, Wien: P.I.E. Peter Lang, 2018, 246 pp., ISBN: 9782807606050, $€ 47.95$.

Cet ouvrage est une version abrégée et remaniée de la thèse de doctorat défendue par Vincent Genin le 18 janvier 2017 à la Faculté de Philosophie et Lettres de l'Université de Liège. Il s'agit d'un travail considérable, qui couvre le parcours de nombreux juristes belges non seulement pendant la période mentionnée dans son titre (1914-1940), mais aussi au-delà, en particulier dans le dernier quart du XIX ${ }^{\text {ème }}$ siècle. La recherche se fonde sur un matériau impressionnant, composé non seulement de publications classiques et d'archives publiques, mais aussi, ce qui confère à la recherche un caractère particulièrement novateur, de correspondances et d'archives privées et du fruit de recherches personnelles diverses. Le travail est divisé suivant un plan chronologique, avec une périodisation en trois temps : la première guerre mondiale (1914-1918), la période 1918-1930 et la période 1930-1940, cette dernière étant plus particulièrement consacrée au cas de Fernand Dehousse. L'objectif est d'étudier la vie et les travaux des internationalistes belges, à la fois en évaluant leur influence dans le cadre de la vie politique belge et en comparant cette influence avec celles qu'ont pu avoir des internationalistes dans d'autres pays. Le propos est généralement clair et fluide, et la langue est soignée.

Tout en reconnaissant ces indéniables qualités, on pourrait regretter le caractère souvent descriptif de nombreux passages di livre. Au-delà de l'affirmation générale d'une étude des juristes belges dans le contexte belge, affirmé en introduction, on a peine à identifier une hypothèse de travail claire. L'introduction se révèle particulièrement peu éclairante, le manque de précision sur ce point fondamental contrastant avec de longs développements abstraits et généraux dont il n'est pas toujours évident de comprendre l'intérêt. Le titre lui-même pourrait donner une indication à cet égard, mais les expressions qui le composent ne sont pas précisées (qu'est-ce qu'un «mythe juridique »? ou un « déclassement international »?), de sorte qu'il est très difficile au lecteur d'évaluer ensuite la démonstration supposée venir à son appui. De démonstration, il ne semble d'ailleurs pas toujours en être question. On a parfois l'impression de découvrir une suite de notes de lecture, opérées à partir de certaines événements (voire anecdotes) qui ont jalonné la vie d'un auteur, de ses écrits ou de ses actions, mais on a peine à comprendre en quoi cette suite a un lien avec une thèse en tant que telle. Les chapitres ou sous-chapitres débutent, se terminent ou se succèdent souvent sans conclusion intermédiaire ou sans

(C) OLIVIER CORTEN, 2019 | DOI:10.1163/15718050-12340107

This is an open access article distributed under the terms of the prevailing CC-BY-NC license

at the time of publication. 
transition susceptibles de permette au lecteur de retrouver le fil rouge supposé relier les différentes étapes du raisonnement. Un exemple caractéristique est le passage du chapitre II au chapitre III, consacré au cas particulier de Fernand Dehousse. A ce stade, on éprouve tout particulièrement des difficultés à percevoir le lien de cette étude biographique avec la thèse, l'auteur ne nous donnant guère d'indication en ce sens.

Un deuxième problème substantiel est celui du caractère limité de la mise en contexte politique. Evaluer le rôle des spécialistes de droit international dans la prise de décision en Belgique aurait supposé de recourir à des outils de sociologie politique pour mesurer le rôle des différents acteurs, le cas échéant dans une perspective inspirée de Bourdieu, comme le suggère l'auteur lui-même dans son introduction. Il est cependant difficile de retrouver trace d'une semblable démarche dans le corps du texte. Ce dernier reste essentiellement composé d'une description du contenu des sources récoltées, mais sans que l'auteur prenne toujours la distance nécessaire pour procéder à une analyse critique de celles-ci. On ne trouvera pas non plus de comparaison aboutie entre les spécialistes de droit international en Belgique et dans d'autres Etats, comparaison qui aurait été susceptible de démontrer, ou au contraire de mettre en cause, une spécificité belge. Certains passages semblent ébaucher une réflexion en ce sens, comme lorsqu'il est affirmé que les «petits pays » seraient plus favorables à la codification que les grandes puissances. Mais cet énoncé n'est jamais explicité ni développé, et on se demande dès lors sur quel fondement il repose, et quelles en seraient les répercussions pour la thèse supposément défendue par l'auteur.

Un troisième problème, lié au précédent, renvoie à certaines difficultés de mise en contexte sur le plan plus strictement juridique. Ici encore, l'auteur relaie des propos ou nous livre ses impressions sans systématiquement faire preuve d'une distance critique, cette fois sur le plan du droit international luimême. Bien entendu, il ne s'agit pas d'une étude «en» droit international, mais « sur » le droit international. Il reste que certaines affirmations paraissent problématiques au regard du cadre de références des internationalistes qui font l'objet de l'étude. Ainsi, et pour ne reprendre qu'un exemple, il est difficile de comprendre comment on peut affirmer à plusieurs reprises que la guerre menée par l'Allemagne contre la Belgique était contraire au droit international, tout en reprenant la thèse répandue (mais excessive) selon laquelle le déclenchement d'une guerre relevait alors d'une prérogative souveraine des Etats.

Au final, au-delà des limites qui viennent d'être évoquées et comme le souligne Emmanuelle Tourme-Jouannet dans sa préface, on reste devant un 
ouvrage particulièrement riche sur le plan empirique, qui ne manquera pas d'attirer utilement l'attention des historiens sur certains parcours singuliers de juristes belges pendant les périodes historiques examinées.

\section{Olivier Corten}

Centre de droit international

Université libre de Bruxelles 